\title{
A note on supersymmetric type II solutions of Lifshitz type
}

\section{Michela Petrini $^{a, b}$ and Alberto Zaffaroni ${ }^{c}$}

${ }^{a}$ Laboratoire de Physique Théorique et Hautes Energies, Université Pierre et Marie Curie, 4 Place Jussieu, 75252 Paris Cedex 05, France

${ }^{b}$ IPhT, CEA Saclay, F-91191 Gif-sur-Yvette, France

${ }^{c}$ Dipartimento di Fisica, Università di Milano-Bicocca and INFN, sezione di Milano-Bicocca, piazza della Scienza 3, Milano 20126, Italy

E-mail: petrini@lpthe.jussieu.fr, alberto.zaffaroni@mib.infn.it

ABSTRACT: We discuss a class of supersymmetric type II non-relativistic solutions with exact or asymptotic scale invariance. As already emerged from previous investigations, we find a clear correspondence between anisotropic $d$-dimensional vacua and relativistic solutions in $d+1$ dimensions. We will show that supersymmetric four-dimensional Poincaré invariant backgrounds in type IIB can descend to analogous solutions with anisotropic scaling in $t$ and $(x, y)$. This result can be applied to scale invariant theories, domain walls interpolating between four-dimensional Lifshitz vacua and more general solutions with only asymptotic, approximate scaling behaviour and hyperscaling violation.

Keywords: Flux compactifications, AdS-CFT Correspondence, Holography and condensed matter physics (AdS/CMT)

ARXIV EPRINT: 1202.5542 


\section{Contents}

1 Introduction 1

2 Non-relativistic solutions in type IIA 4

2.1 Bianchi identities and equations of motion 5

2.2 Supersymmetry conditions 6

2.3 Summary: from four to three dimensions 8

3 Non-relativistic solutions in type IIB $\quad 9$

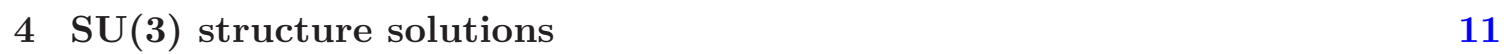

$\begin{array}{ll}4.1 \text { Examples } & 12\end{array}$

$\begin{array}{lll}\text { 4.1.1 } & \text { Lif }_{4} \text { from } A d S_{5} \text { solutions } & 13\end{array}$

$\begin{array}{lll}\text { 4.1.2 Asymptotically } \text { Lif }_{4} \text { solutions } & 14\end{array}$

$\begin{array}{lll}\text { 4.1.3 Solutions with hyperscaling violation } & 16\end{array}$

$\begin{array}{lll}5 & \text { Conclusions } & 17\end{array}$

$\begin{array}{lr}\text { A Notations and useful formulae } & 18\end{array}$

$\begin{array}{ll}\text { B The conditions for supersymmetry } & 19\end{array}$

\section{Introduction}

The gauge/gravity correspondence relates relativistic strongly coupled gauge theories to supergravity on Anti de Sitter or warped Minkowski space-times. Recently, the application of the ideas of holography to condensed matter systems have motivated the study of non relativistic geometries in string/supergravity theories. For instance, systems of strongly correlated electrons can exhibit critical points with an anisotropic rescaling between time and space

$$
t \rightarrow \lambda^{z} t \quad x^{i} \rightarrow \lambda x^{i} \quad i=1 \ldots D .
$$

According to the holographic dictionary such behaviour should be described by a Lifshitz geometry

$$
\mathrm{d} s^{2}=-r^{2 z} \mathrm{~d} t^{2}+r^{2} \sum_{i=1}^{D}\left(\mathrm{~d} x^{i}\right)^{2}+\frac{\mathrm{d} r^{2}}{r^{2}},
$$

where $r$ is the holographic energy direction.

Contrary to other non-relativistic solutions, like Schrödinger geometries, embedding Lifshitz solutions in string theory turned out to be a relatively non trivial issue. Lifshitz geometries appear in four dimensional models of gravity coupled to a topological term 
or a massive vector $[1,2]$. While initially some no-go theorems seemed to exclude the possibility of embedding Lifshitz solutions in the full supergravity theory, ${ }^{1}$ explicit examples of 10-dimensional solutions with a Lifshitz factor were constructed in [5] and subsequently generalised in [6-8], and, in the context of $\mathcal{N}=2$ gauged supergravity, in [9, 10].

The four-dimensional Lifshitz solutions found in [6] are supersymmetric and have scaling exponent $z=2$. They can be obtained as circle reductions of Schrödinger solutions with $z=0$, which represent a plane wave propagating on the world-volume of D3-branes transverse to a Ricci-flat space and with non-zero RR and NS magnetic 3-form flux. The examples found in [6] can be thought of as a reduction of an $A d S_{5}$ type IIB vacua with the addition of some fluxes. A similar result has been proven in [10] at the level of gauge supergravity: any $(d+1)$-dimensional gauge supergravity admitting $A d S_{d+1}$ vacua gives, upon reduction on a circle, a $d$-dimensional gauge supergravity admitting $L i f_{d}$ vacua with $z=2$. As we show below a similar result can also be obtained at the level of vacua in the 10-dimensional type II supergravities, avoiding all complications related to truncations and dimensional reductions.

In this note in fact we analyse in detail the supersymmetry constraints on a large class of non-relativistic solutions which include and generalise the examples found in [6]. We consider type II solutions corresponding to three-dimensional theories with anisotropic scaling in time $t$ and space $(x, y)$. This class of solutions will include scale invariant theories, domain walls interpolating between four-dimensional Lifshitz vacua and more general solutions with only asymptotic, approximate scaling behaviour or hyperscaling violation. We will show that many supersymmetric four-dimensional Poincaré invariant backgrounds in type IIB descend to analogous solutions with anisotropic scaling in $t$ and $(x, y)$. This result strengthens the correspondence between $A d S_{5}$ and $L i f_{4}$ vacua found in [6, 10]. In particular, starting from supersymmetric domain walls in type IIB interpolating between $A d S_{5}$ vacua, we often find solutions interpolating between the corresponding $L_{i f} f_{4}$ vacua.

More generally we provide a framework for constructing supersymmetric nonrelativistic backgrounds which can be useful also to study solutions with only asymptotic scaling and different IR behaviour, corresponding for example to confining solutions. We hope to report on the subject in the next future.

For convenience, in the rest of the Introduction, we summarise the relevant facts we need about supersymmetry. In order to study non relativistic supersymmetric vacua we will use the formalism of Generalised Complex Geometry $[11,12]$. In the case where the space is the (warped product)

$$
\mathrm{d} s^{2}=e^{2 A} \mathrm{~d} s_{4}^{2}+\mathrm{d} s_{6}^{2}
$$

of four-dimensional Minkowski or Anti de Sitter times an internal six-dimensional manifold $M_{6}$, the supersymmetry variations can be re-expressed as a set of differential equations on the internal manifold. More precisely, if $\eta_{+}^{1}$ and $\eta_{+}^{2}$ are two chiral spinors on $M_{6}$, we can define the bispinors

$$
\Phi_{ \pm}=\eta_{+}^{1} \eta_{ \pm}^{2 \dagger}
$$

\footnotetext{
${ }^{1}$ See for example $[3,4]$.
} 
which, by Fierz identity, can be seen as sums of even, $\Phi_{+}$, and odd, $\Phi_{-}$, forms on $M_{6}{ }^{2}$ The supersymmetry variations can be shown to be equivalent to the following set of differential equations ${ }^{3}$

$$
\begin{aligned}
\mathrm{d}_{H}\left(e^{2 A-\phi} \Phi_{1}\right) & =0, \\
\mathrm{~d}_{H}\left(e^{A-\phi} \operatorname{Im} \Phi_{2}\right) & =0, \\
\mathrm{~d}_{H}\left(e^{3 A-\phi} \operatorname{Re} \Phi_{2}\right) & =\frac{1}{8} e^{4 A} * \lambda(F),
\end{aligned}
$$

where $F$ is the sum of the RR fluxes on the internal manifold, $H$ is the NS flux, $\Phi_{1}=\Phi_{+}$ and $\Phi_{2}=\Phi_{-}$in IIA while for IIB $\Phi_{1}=\Phi_{-}$and $\Phi_{2}=\Phi_{+}[13,14]$.

A similar approach has been very recently used to study generic ten-dimensional vacua [15]. In this case, the conditions for supersymmetry can be reformulated in terms of intrinsic objects constructed with the type II supersymmetry parameters: a ten-dimensional "spinor" $\Phi$

$$
\Phi=\epsilon_{1} \bar{\epsilon}_{2}
$$

and two one-forms $K_{1}$ and $K_{2}$

$$
K_{1 M}=\frac{1}{32} \bar{\epsilon}_{1} \Gamma_{M} \epsilon_{1}, \quad K_{2 M}=\frac{1}{32} \bar{\epsilon}_{2} \Gamma_{M} \epsilon_{2},
$$

which annihilate the supersymmetry parameters ${ }^{4}$

$$
K_{1} \cdot \epsilon_{1}=K_{2} \cdot \epsilon_{2}=0 .
$$

Each $K_{i}$ is null and gives rise to a basis of vielbeine $\left(e_{-i} \equiv K_{i}, e_{+i}, e_{I}\right)$ which we normalize as

$$
e_{-i} \cdot e_{+i}=\frac{1}{2}
$$

In this formalism the supersymmetry conditions are [15]

$$
\begin{gathered}
\mathrm{d}_{H}\left(e^{-\phi} \Phi\right)=-\left(\tilde{K} \wedge+\iota_{K}\right) F \\
\mathrm{~d} \tilde{K}=\iota_{K} H \\
\left(e_{+1} \cdot \Phi \cdot e_{+2}, \Gamma^{M N}\left[ \pm \mathrm{d}_{H}\left(e^{-\phi} \Phi \cdot e_{+2}\right)+e^{\phi} \mathrm{d}^{\dagger}\left(e^{-2 \phi} e_{+2}\right) \Phi-F\right]\right) \\
\left(e_{+1} \cdot \Phi \cdot e_{+2},\left[\mathrm{~d}_{H}\left(e^{-\phi} e_{+1} \cdot \Phi\right)-e^{\phi} \mathrm{d}^{\dagger}\left(e^{-2 \phi} e_{+2}\right) \Phi-F\right] \Gamma^{M N}\right),
\end{gathered}
$$

plus the condition $L_{K} g=0$. The vectors $K$ and $\tilde{K}$ are

$$
K=\frac{K_{1}+K_{2}}{2} \quad \tilde{K}=\frac{K_{1}-K_{2}}{2} .
$$

In (1.12) and (1.13) we use the ten dimensional Mukai pairing $(A, B)=(A \wedge \lambda(B))_{10}$ and, in (1.12), the upper sign is for IIA and the lower is for IIB.

\footnotetext{
${ }^{2}$ On $T\left(M_{6}\right) \oplus T^{*}\left(M_{6}\right)$, the polyforms $\Phi_{+}$and $\Phi_{-}$correspond to pure spinors of positive and negative chirality, respectively.

${ }^{3}$ Here $_{H}=(\mathrm{d}-H \wedge)$ and $\lambda\left(C_{p}\right)=(-1)^{[p / 2]} C_{p}$ if $C_{p}$ is a form of degree $p$.

${ }^{4} K_{i} \cdot \epsilon_{i}=K_{i} \Gamma^{M} \epsilon_{i}$ is the Clifford multiplication.
} 
In this paper we will apply this formalism to the study of non-relativistic solutions of Lifshitz type in type II supergravity. In section 2 we discuss a class of non relativistic solutions of type IIA, including and generalizing [6], and we reduce the supersymmetry constraints to those of an auxiliary four-dimensional Poincaré invariant vacuum of type IIB plus a set of algebraic and differential constraints on the fluxes. For reader convenience, we summarise our findings in section 2.3. In section 3 we discuss the parallel case of non relativistic type IIB solutions. In section 4 we discuss the particular case of SU(3) structures and we provide examples of $\mathrm{Lif}_{4}$ solutions, domain walls and backgrounds with hyperscaling violation.

\section{Non-relativistic solutions in type IIA}

We look for static solutions in IIA supergravity corresponding to three-dimensional theories with anisotropic scaling in time $t$ and space $(x, y)$. We do not require to have exact scaling symmetry in order to accommodate also domain wall solutions interpolating between four-dimensional Lifshitz vacua and more general solutions with only asymptotic or approximate scaling behaviour. As usual, one of the transverse directions will play the role of the radial coordinate.

On the basis of the existing examples of Lifshitz solutions [6] and of the general structure of supersymmetric solutions in IIA [15], we choose a metric of the form

$$
\mathrm{d} s_{10}^{2}=-e^{2 A_{1}} \mathrm{~d} t^{2}+e^{2 A_{2}}\left(\mathrm{~d} x^{2}+\mathrm{d} y^{2}\right)+\left(e^{1}\right)^{2}+\mathrm{d} s_{6}^{2}
$$

with

$$
q e^{1}=\mathrm{d} \varphi+\mu,
$$

where $\varphi$ denotes a generic angular direction, $\mu$ is a connection on $M_{6}$ with curvature $\alpha \equiv \mathrm{d} \mu$, and $A_{1}, A_{2}$ and $q$ are functions on $M_{6}$.

According to this splitting we assume the following structure for the fluxes ${ }^{5}$

$$
\begin{aligned}
H^{I I A} & =h+\mathrm{d}\left(e^{01}\right), \\
F^{I I A} & =-q\left(e^{1} f+e^{0 x y} * \lambda(f)\right)+\left(1+e^{01}\right)\left(w+e^{x y} * \lambda(w)\right),
\end{aligned}
$$

where we defined the three-dimensional vielbeine

$$
e^{0}=e^{A_{1}} \mathrm{~d} t, \quad e^{x}=e^{A_{2}} \mathrm{~d} x, \quad e^{y}=e^{A_{2}} \mathrm{~d} y,
$$

and $f$ and $w$ denote formal sums of odd and even forms on the internal space $M_{6}$,

$$
\begin{aligned}
f & =f_{1}+f_{3}+f_{5}, \\
w & =w_{0}+w_{2}+w_{4}+w_{6} .
\end{aligned}
$$

\footnotetext{
${ }^{5}$ The RR fluxes are written in the democratic formulation [16] as a formal sum $F=\sum_{k=0}^{5} F_{(2 k)}$, with $k$ even in IIA and half-integer in IIB, subject to the condition $F=*_{10} \lambda(F)$. We have inherited from the democratic approach a somehow unnatural convention for the star product: $* C \wedge C=|C|^{2}$ Vol.
} 
All star products are taken on the internal space $M_{6}$. For simplicity, we omit the wedge products and define $e^{a b c \cdots}=e^{a} \wedge e^{b} \wedge e^{c} \wedge \cdots$. Also, for notational convenience, we write the type IIA dilaton $\phi_{A}$ in the form

$$
e^{-\phi_{A}}=q e^{-\phi}
$$

The splitting of the metric is suggested by the conditions for supersymmetry. From (1.10)-(1.13) it follows that $K$ must be dual to a Killing vector. For the static solutions we are considering we have a natural Killing vector corresponding to time translations. It is then natural to identify $K=e^{0}$. The choice of metric (2.1) corresponds to the general class of solutions where the second vector identifies a special direction in the transverse space, $\tilde{K}=e^{1}$.

The ansatz for the metric and fluxes makes it possible to reduce the search for vacua to a purely six-dimensional problem. The equations of motion and the Bianchi identities for the fluxes reduce to a set of differential constraints for the forms $(\alpha, w, h, f)$ on $M_{6}$ and the supersymmetry conditions can be written in terms of the six-dimensional pure spinors (1.4). One of the main results of our analysis is that the six-dimensional data $\left(\phi, h, f, \Phi_{ \pm}\right)$must satisfy the conditions for supersymmetry of a four-dimensional Poincaré invariant vacuum of type IIB, (1.5). This will allow to construct non-relativistic solutions with three space-time dimensions starting from known four-dimensional vacua. In the following sections we analyse the equations of motion and the supersymmetry constraints. We have summarised the results in section 2.3.

\subsection{Bianchi identities and equations of motion}

The fluxes have to satisfy the ten dimensional equations of motion and Bianchi identities

$$
\begin{aligned}
\mathrm{d} F^{\mathrm{IIA}}-H^{\mathrm{IIA}} \wedge F^{\mathrm{IIA}} & =0, \\
\mathrm{~d} H^{I I A} & =0 \\
\mathrm{~d}\left(e^{\left.-2 \phi_{I I A} * H^{I I A}\right)}\right. & =-\left.\frac{1}{2} F^{I I A} \wedge * F^{I I A}\right|_{8} .
\end{aligned}
$$

The previous conditions reduce to the following constraints on the internal forms $h$ and $f$

$$
\begin{aligned}
\mathrm{d}_{h} f & =0, \\
\mathrm{~d}_{h}\left(q e^{A_{1}+2 A_{2}} * \lambda(f)\right) & =0, \\
\mathrm{~d} h & =0, \\
\mathrm{~d}\left(q e^{A_{1}+2 A_{2}-2 \phi} * h\right) & =\left.q e^{A_{1}+2 A_{2}} f * f\right|_{4},
\end{aligned}
$$

and for the forms $w$ and $\alpha$

$$
\begin{aligned}
\mathrm{d}_{h} w & =\alpha f, \\
\mathrm{~d}_{h}\left(e^{2 A_{2}} * \lambda(w)\right) & =0, \\
\mathrm{~d} \alpha & =0, \\
\mathrm{~d}\left(e^{2 A_{2}-2 \phi} * \alpha\right) & =-\left.e^{2 A_{2}} f * w\right|_{5},
\end{aligned}
$$


plus the the algebraic relation

$$
e^{-2 \phi} \alpha * h=\left.w * f\right|_{5}
$$

and the second order differential equation for $q$

$$
* \mathrm{~d}\left(q e^{2 A_{2}+A_{1}-2 \phi} * \mathrm{~d}\left(q e^{-A_{1}}\right)\right)=e^{2 A_{2}}\left(e^{-2 \phi}|\alpha|^{2}+|w|^{2}\right) .
$$

\subsection{Supersymmetry conditions}

The supersymmetry conditions (1.10)-(1.13) can also be reduced to a set of equations for the forms on $M_{6}$. We will first split the ten-dimensional spinors into the product of a twodimensional and an eight-dimensional spinor, and then further reduce the eight-dimensional spinors to six-dimensional ones. The form (2.1) of the metric suggests to choose $e^{0}$ and $e^{1}$ as preferred directions for our basis of (real) ten-dimensional gamma matrices

$$
\begin{aligned}
& \Gamma^{0}=i \sigma_{2} \otimes \mathbb{I}_{(8)}, \\
& \Gamma^{1}=\sigma_{1} \otimes \mathbb{I}_{(8)}, \\
& \Gamma^{I}=\sigma_{3} \otimes \hat{\gamma}^{I} \quad I=1, \ldots, 8 .
\end{aligned}
$$

In type IIA the ten dimensional spinors $\epsilon_{1}$ and $\epsilon_{2}$ are Majorana-Weyl and have opposite chirality. Using the fact that $K=e^{0}$ and $\tilde{K}=e^{1}$ and the choice (2.16) of gamma matrices, the conditions (1.8) become

$$
\begin{aligned}
& \left(\Gamma^{0}+\Gamma^{1}\right) \epsilon_{1}=0, \\
& \left(\Gamma^{0}-\Gamma^{1}\right) \epsilon_{2}=0,
\end{aligned}
$$

and imply

$$
\epsilon_{1}=\left(\begin{array}{l}
1 \\
0
\end{array}\right) \hat{\eta}_{1} \quad \epsilon_{2}=\left(\begin{array}{l}
0 \\
1
\end{array}\right) \hat{\eta}_{2},
$$

where the eight-dimensional spinors $\hat{\eta}_{1}$ and $\hat{\eta}_{2}$ are real and have both positive chirality. ${ }^{6}$ We further split the eight transverse directions into two plus six. For the gamma matrices this gives

$$
\begin{aligned}
\hat{\gamma}^{x} & =\sigma_{1} \otimes \mathbb{I}, \\
\hat{\gamma}^{y} & =\sigma_{3} \otimes \mathbb{I}, \\
\hat{\gamma}^{m} & =\sigma_{2} \otimes \gamma^{m} \quad m=1, \ldots, 6 .
\end{aligned}
$$

The spinors split accordingly

$$
\hat{\eta}_{i}=\sqrt{\frac{C}{2}}\left[\left(\begin{array}{c}
1 \\
i
\end{array}\right) \eta_{+}^{i}+\left(\begin{array}{c}
1 \\
-i
\end{array}\right) \eta_{-}^{i}\right] \quad i=1,2,
$$

${ }^{6}$ The ten-dimensional and the six-dimensional chiral gammas are

$$
\Gamma_{10}=\Gamma^{0} \ldots \Gamma^{9}=\sigma_{3} \otimes \hat{\gamma}_{9} \quad \gamma_{7}=-i \gamma_{1} \cdots \gamma_{6} .
$$


where $\eta_{+}^{i}$ are six-dimensional spinors of positive chirality and $\eta_{-}=\left(\eta_{+}\right)^{*}$. It is convenient to redefine $\hat{\eta}_{1} \rightarrow \hat{\gamma}^{x y} \hat{\eta}_{1}$ or, equivalently, $\eta_{1} \rightarrow-i \eta_{1}$.

By construction the vectors $K_{1}$ and $K_{2}$ are given by

$$
\begin{aligned}
& K_{(1) M}=\frac{1}{32} \bar{\epsilon}_{1} \Gamma_{M} \epsilon_{1}=\frac{C}{16}\left\|\eta_{+}^{1}\right\|^{2}(1,1,0,0, \ldots, 0)=\frac{C}{16}\left\|\eta_{+}^{1}\right\|^{2}\left(e^{0}+e^{1}\right)_{M}, \\
& K_{(2) M}=\frac{1}{32} \bar{\epsilon}_{2} \Gamma_{M} \epsilon_{2}=\frac{C}{16}\left\|\eta_{+}^{2}\right\|^{2}(1,-1,0,0, \ldots, 0)=\frac{C}{16}\left\|\eta_{+}^{2}\right\|^{2}\left(e^{0}-e^{1}\right)_{M} .
\end{aligned}
$$

For simplicity, we restrict to the case where the norms of the six-dimensional spinors are equal: $\left\|\eta_{+}^{1}\right\|=\left\|\eta_{+}^{2}\right\|=\left\|\eta_{+}\right\|$. We expect that, analogously to relativistic vacua, this is the case of interest for backgrounds generated by D-branes. With equal norm for the spinors, the form of $K$ and $\tilde{K}$ is particularly simple,

$$
K=\frac{C}{16}\left\|\eta_{+}\right\|^{2} e^{0} \quad \tilde{K}=\frac{C}{16}\left\|\eta_{+}\right\|^{2} e^{1} .
$$

We still need to require that $K$ is Killing. This fixes the functional dependence of $C$

$$
C=16 \frac{e^{A_{1}}}{\left\|\eta_{+}\right\|^{2}}
$$

and we finally have

$$
K=e^{A_{1}} e^{0} \quad \tilde{K}=e^{A_{1}} e^{1} .
$$

The normalization of $C$ is fixed in such a way that $K$ is precisely dual to $\frac{\partial}{\partial t}$.

The ten dimensional "pure-spinor" also factorises into the product of four and sixdimensional forms

$$
\Phi=\epsilon_{1} \bar{\epsilon}_{2}=-\frac{1}{2}\left(1+e^{01}\right) \Phi_{(8)},
$$

with

$$
\Phi_{(8)}=\frac{16}{\left\|\eta_{+}\right\|^{2}} e^{A_{1}}\left\{\operatorname{Im}\left[\left(1+i e^{x y}\right) \Phi_{+}\right]-\operatorname{Re}\left[\left(e^{x}-i e^{y}\right) \Phi_{-}\right]\right\} .
$$

$\Phi_{ \pm}$are the six-dimensional pure spinors defined in (1.4)

$$
\Phi_{+}=\eta_{+}^{1} \eta_{+}^{2 \dagger}, \quad \Phi_{-}=\eta_{+}^{1} \eta_{-}^{2 \dagger} .
$$

Let us now consider the supersymmetry conditions (1.10)-(1.13). Equation (1.11) is trivially satisfied. From equation (1.10) we obtain the six-dimensional equations

$$
\begin{aligned}
\mathrm{d}_{h}\left(q e^{A_{1}-\phi} \frac{1}{\left\|\eta_{+}\right\|^{2}} \operatorname{Im} \Phi_{+}\right) & =0, \\
\mathrm{~d}_{h}\left(q e^{A_{1}+2 A_{2}-\phi} \frac{1}{\left\|\eta_{+}\right\|^{2}} \operatorname{Re} \Phi_{+}\right) & =\frac{q}{8} e^{A_{1}+2 A_{2}} * \lambda(f), \\
\mathrm{d}_{h}\left(q e^{A_{1}+A_{2}-\phi} \frac{1}{\left\|\eta_{+}\right\|^{2}} \Phi_{-}\right) & =0 .
\end{aligned}
$$

Notice that on the right hand side of equation (1.10) $w$ disappears

$$
\left(\tilde{K} \wedge+\iota_{K}\right) F=q e^{A_{1}}\left(1+e^{01}\right) \wedge e^{x y} * \lambda(f) .
$$


The conditions (1.12) and (1.13) can also be simplified. The computation is more involved and we give it in appendix B. What is important for our analysis is that they reduce to two independent sets of conditions, one for $f$ and one for $\alpha$ and $w$. All conditions for $f$ are automatically satisfied once we fix

$$
e^{A_{1}}=\frac{e^{2 A_{2}}}{q},
$$

while the conditions for $\alpha$ and $w$ are

$$
\begin{aligned}
\operatorname{Im}\left[\left(\bar{\Phi}_{+}, \frac{e^{-\phi}}{\left\|\eta_{+}\right\|^{2}} \alpha \cdot \Phi_{+}\right)_{6}\right] & =\operatorname{Im}\left[\left(\bar{\Phi}_{+}, w\right)_{6}\right]=0 \\
\operatorname{Re}\left[\left(\bar{\Phi}_{+},\left(\frac{e^{-\phi}}{\left\|\eta_{+}\right\|^{2}} \alpha \cdot \Phi_{+}-i w\right)\right)_{6}\right] & =\operatorname{Re}\left[\left(\bar{\Phi}_{+},\left(\frac{e^{-\phi}}{\left\|\eta_{+}\right\|^{2}} \Phi_{+} \cdot \alpha+i w\right)\right)_{6}\right]=0 \\
\operatorname{Im}\left[\left(\bar{\Phi}_{+}, \gamma^{m n}\left(\frac{e^{-\phi}}{\left\|\eta_{+}\right\|^{2}} \alpha \cdot \Phi_{+}-i w\right)\right)_{6}\right] & =\operatorname{Im}\left[\left(\bar{\Phi}_{+},\left(\frac{e^{-\phi}}{\left\|\eta_{+}\right\|^{2}} \Phi_{+} \cdot \alpha+i w\right) \gamma^{m n}\right)_{6}\right]=0, \\
\left(\Phi_{-}, \gamma^{n}\left(\frac{4 e^{-\phi}}{\left\|\eta_{+}\right\|^{2}} \alpha \wedge \Phi_{+}-i w\right)\right)_{6} & =\left(\Phi_{-},\left(\frac{4 e^{-\phi}}{\left\|\eta_{+}\right\|^{2}} \alpha \wedge \bar{\Phi}_{+}-i w\right) \gamma^{n}\right)_{6}=0,
\end{aligned}
$$

where $\cdot$ denote the Clifford product and $(A, B)_{6}=(A \wedge \lambda(B))_{6}$ is the six-dimensional Mukai pairing.

With the redefinition (2.34) the conditions (2.12) and (2.30)-(2.32) involving $(f, h, \phi)$ become identical to the equations for the fluxes and the supersymmetry conditions for a type IIB vacuum with four-dimensional Poincaré invariance. This is one of the advantages of our formalism: the fields $(f, h, \phi)$ in our non-relativistic solutions are the same as for four-dimensional relativistic solutions, where many supersymmetric backgrounds are known. In the next section we summarise our findings.

\subsection{Summary: from four to three dimensions}

Consider a supersymmetric type IIB background

$$
\mathrm{d} s_{10}^{2}=e^{2 A}\left(\eta_{\mu \nu} \mathrm{d} x^{\mu} \mathrm{d} x^{\nu}\right)^{2}+\mathrm{d} s_{6}^{2}, \quad \mu=0, \cdots, 3,
$$

with dilaton $\phi$ and fluxes defined on $M_{6}$

$$
H_{I I B}=h, \quad F_{I I B}=f+e^{0 x y z} * \lambda(f),
$$

and satisfying the Bianchi identities and the equations of motion

$$
\begin{aligned}
\mathrm{d}_{h} f & =0, \\
\mathrm{~d}_{h}\left(e^{4 A} * \lambda f\right) & =0, \\
\mathrm{~d} h & =0, \\
\mathrm{~d}\left(e^{4 A-2 \phi} * h\right) & =\left.e^{4 A} f * f\right|_{4} .
\end{aligned}
$$

The type IIB supersymmetry parameters decompose as

$$
\epsilon_{i}=\zeta_{+} \eta_{+}^{i}+\zeta_{-} \eta_{-}^{i} \quad i=1,2
$$


where $\zeta_{+}$are four-dimensional Weyl spinors and $\eta_{+}^{i}$ are six-dimensional spinors on $M_{6}$ normalized as

$$
\left\|\eta_{+}^{1}\right\|=\left\|\eta_{+}^{2}\right\|=\frac{c_{+}}{2} e^{A} .
$$

The conditions for supersymmetry can be written in terms of the six-dimensional pure spinors, $\Phi_{ \pm}$of (1.4), as

$$
\begin{aligned}
\mathrm{d}_{h}\left(e^{A-\phi} \operatorname{Im} \Phi_{+}\right) & =0, \\
\mathrm{~d}_{h}\left(e^{3 A-\phi} \operatorname{Re} \Phi_{+}\right) & =\frac{c_{+}}{16} e^{4 A} * \lambda f, \\
\mathrm{~d}_{h}\left(e^{2 A-\phi} \Phi_{-}\right) & =0 .
\end{aligned}
$$

Given such a four-dimensional supersymmetric type IIB vacuum, we can construct a non-relativistic supersymmetric solution in type IIA with metric (2.1) and

$$
e^{A_{1}}=\frac{e^{2 A}}{q}, \quad e^{A_{2}}=e^{A}, \quad e^{\phi_{A}}=\frac{e^{\phi}}{q},
$$

provided we find on $M_{6}$ a two-form $\alpha$ and a polyform $w=\sum_{k=0}^{3} w_{2 k}$ satisfying the equations (coming from the 10-dimensional Bianchi identities and equations of motion)

$$
\begin{aligned}
\mathrm{d}_{h} w & =\alpha f, \\
\mathrm{~d}_{h}\left(e^{2 A} * \lambda(w)\right) & =0, \\
\mathrm{~d} \alpha & =0, \\
\mathrm{~d}\left(e^{2 A-2 \phi} * \alpha\right) & =-\left.e^{2 A} f * w\right|_{5}, \\
e^{-2 \phi} \alpha * h & =\left.w * f\right|_{5},
\end{aligned}
$$

and the supersymmetry constraints

$$
\begin{aligned}
\operatorname{Im}\left[\left(\bar{\Phi}_{+}, e^{-\phi-A} \alpha \cdot \Phi_{+}\right)_{6}\right] & =\operatorname{Im}\left[\left(\bar{\Phi}_{+}, w\right)_{6}\right]=0 \\
\operatorname{Re}\left[\left(\bar{\Phi}_{+},\left(e^{-\phi-A} \frac{2}{c_{+}} \alpha \cdot \Phi_{+}-i w\right)\right)_{6}\right] & =\operatorname{Re}\left[\left(\bar{\Phi}_{+},\left(e^{-\phi-A} \frac{2}{c_{+}} \Phi_{+} \cdot \alpha+i w\right)\right)_{6}\right]=0, \\
\operatorname{Im}\left[\left(\bar{\Phi}_{+}, \gamma^{m n}\left(e^{-\phi-A} \frac{2}{c_{+}} \alpha \cdot \Phi_{+}-i w\right)\right)_{6}\right] & =\operatorname{Im}\left[\left(\bar{\Phi}_{+},\left(e^{-\phi-A} \frac{2}{c_{+}} \Phi_{+} \cdot \alpha+i w\right) \gamma^{m n}\right)_{6}\right]=0, \\
\left(\Phi_{-}, \gamma^{n}\left(e^{-\phi-A} \frac{8}{c_{+}} \alpha \wedge \Phi_{+}-i w\right)\right)_{6} & =\left(\Phi_{-},\left(e^{-\phi-A} \frac{8}{c_{+}} \alpha \wedge \bar{\Phi}_{+}-i w\right) \gamma^{n}\right)_{6}=0,
\end{aligned}
$$

where $\cdot$ denote the Clifford product and $(A, B)_{6}=(A \wedge \lambda B)_{6}$ is the six-dimensional Mukai pairing. The function $q$ is determined by the second order differential equation

$$
* \mathrm{~d}\left(e^{4 A-2 \phi} * \mathrm{~d}\left(q^{2} e^{-2 A}\right)\right)=e^{2 A}\left(e^{-2 \phi}|\alpha|^{2}+|w|^{2}\right) .
$$

\section{Non-relativistic solutions in type IIB}

The situation in type IIB is similar and we will be brief. Many solutions can be obtained by a plain T-duality. However there are a few interesting features of the solutions that deserve comments. We are interested in metrics of the form

$$
\mathrm{d} s_{10}^{2}=-2 q e^{A_{1}} \mathrm{~d} t \mathrm{~d} \varphi+e^{2 A_{2}}\left(\mathrm{~d} x^{2}+\mathrm{d} y^{2}\right)+q^{2} \mathrm{~d} \varphi^{2}+\mathrm{d} s_{6}^{2},
$$


where, as before, $A_{1}, A_{2}$ and $q$ are functions on $M_{6}$. We introduce the null vielbeine

$$
e^{-}=e^{A_{1}}\left(e^{0}+e^{1}\right), \quad e^{+}=-\frac{1}{4} e^{-A_{1}}\left(e^{0}-e^{1}\right),
$$

in terms of

$$
e^{0}=e^{A_{1}} \mathrm{~d} t, \quad e^{1}=q \mathrm{~d} \varphi-e^{A_{1}} \mathrm{~d} t .
$$

Notice that the Killing vector $\frac{\partial}{\partial t}$ is now null and dual to $e^{-}=q e^{A_{1}} \mathrm{~d} \varphi$.

The fluxes are

$$
\begin{aligned}
& H^{I I B}=h-\frac{e^{-A_{1}}}{q} e^{-} \alpha, \\
& F^{I I B}=f+2 e^{+-x y} * \lambda(f)+\frac{e^{-A_{1}}}{q} e^{-}\left(w+e^{x y} * \lambda(w)\right),
\end{aligned}
$$

where we introduced formal sums $f$ and $w$ of odd and even forms defined on the internal space $M_{6}$ as in equations (2.6) and (2.7). Again all star products are taken in $M_{6}$.

Since the ten-dimensional supersymmetry parameters in type IIB have the same chirality, we now have

$$
\epsilon_{1}=\left(\begin{array}{l}
1 \\
0
\end{array}\right) \hat{\eta}_{1} \quad \epsilon_{2}=-\left(\begin{array}{l}
1 \\
0
\end{array}\right) \hat{\eta}_{2},
$$

where the eight-dimensional spinors $\hat{\eta}_{1}$ and $\hat{\eta}_{2}$ are real and both with positive chirality. The sign in $\epsilon_{2}$ is chosen for convenience. We can still split the eight-dimensional spinors in two plus six as in equation (2.22). However now $K_{1}=K_{2}$, so that $\tilde{K}=0$ and $K$ is null

$$
K=e^{-}
$$

Moreover, the ten-dimensional "pure-spinor" is now proportional to $e^{-}$

$$
\Phi=\epsilon_{1} \bar{\epsilon}_{2}=-\frac{1}{2}\left(e^{0}+e^{1}\right) \Phi_{(8)} .
$$

Exactly as before, given a four-dimensional supersymmetric type IIB vacuum corresponding to the pure spinors $\Phi_{ \pm}$, with metric (2.39), dilaton $\phi$ and fluxes $(h, f)$, we can construct a non-relativistic supersymmetric solution with metric (3.1) with

$$
e^{A_{1}}=\frac{e^{2 A}}{q}, \quad e^{A_{2}}=e^{A}, \quad e^{\phi_{B}}=e^{\phi},
$$

provided we find $M_{6}$ forms $\alpha, w$ satisfying the constraints (2.48)-(2.57).

There are a couple of interesting observations to be made. Since $K$ is null in type IIB, the equations of motion are not necessarily a consequence of the supersymmetry conditions. As discussed in [17], all Einstein and dilaton equations of motion follow from supersymmetry except perhaps the $(0, M \neq 0)$ components of the Einstein equations. Indeed, in our case the $(0, M \neq 0)$ components of the Einstein equations imply the conditions (2.14) and (2.15) which are not consequences of supersymmetry nor the Bianchi identities and equations of motion for the fluxes. 
It is interesting to notice also that both sides of equation (1.10) are proportional to $e^{-}$and therefore to $\mathrm{d} \varphi$. This allows to consider more general solutions where some of the functions and fluxes depend on $\varphi$. Examples of this type have been considered in $[5,6,18,19]$. The generalization of our formalism to this case is straightforward. In this paper we consider the simplest example of such construction, obtained by sending

$$
\eta_{+}^{i} \rightarrow e^{i k \varphi / 2} \eta_{+}^{i}
$$

Since $\Phi$ is proportional to $e^{-}$, equation (1.10) is still satisfied. As discussed in appendix B, new terms appear instead in equations (1.12) and (1.13). As a result, in the supersymmetry conditions (2.35)-(2.37) we must replace

$$
\frac{\alpha}{4} \cdot \Phi_{+} \rightarrow \frac{\alpha}{4} \cdot \Phi_{+}+i \frac{k}{2} \Phi_{+}, \quad \Phi_{+} \cdot \frac{\alpha}{4} \rightarrow \Phi_{+} \cdot \frac{\alpha}{4}-i \frac{k}{2} \Phi_{+} \cdot
$$

We will discuss examples of this type in section 4 .

\section{$4 \mathrm{SU}(3)$ structure solutions}

In the previous section we showed how, given a supersymmetric type IIB solution with four-dimensional Poincaré invariance (2.39) and (2.40), one can construct solutions, both in type IIA and type IIB supergravity, with a non-relativistic three-dimensional factor. In this section we will discuss some explicit examples of such construction. We will focus on a class of solutions where the internal manifold $M_{6}$ has $\mathrm{SU}(3)$ structure, since in this case the supersymmetry conditions for the forms $\alpha$ and $w$ take a particularly simple form. We leave the study of $\mathrm{SU}(2)$ structure solutions for a future work.

In rewriting the supersymmetry variations in terms of pure spinors on the internal manifold $M_{6}$, we make the assumption that the six-dimensional supersymmetry parameters $\eta_{+}^{1}$ and $\eta_{+}^{2}$ are globally defined. Generically, two globally defined spinors reduce the structure group of $M_{6}$ to $\mathrm{SU}(2)$. However if they are parallel

$$
\eta^{1}=\eta_{+}, \quad \eta_{+}^{2}=e^{i \theta} \eta_{+},
$$

the structure group is $\mathrm{SU}(3)$. In six dimensions any spinor $\eta_{+}$is annihilated by three gamma matrices

$$
\left(\gamma^{m}+i \gamma^{m+3}\right) \eta_{+}=0, \quad m=1,2,3,
$$

and defines a complex structure. The SU(3) structure can then be equivalently expressed in terms of a $(1,1)$ (with respect to the complex structure) two-form $J$ and a $(3,0)$ three-form $\Omega$, and the pure spinors (1.4) have the form

$$
\Phi_{+}=\frac{1}{8} e^{-i \theta}\left\|\eta_{+}\right\|^{2} e^{-i J}, \quad \Phi_{-}=-\frac{i}{8} e^{i \theta}\left\|\eta_{+}\right\|^{2} \Omega .
$$

In ordinary four-dimensional vacua, the case $\theta=0$ corresponds to a manifold $M_{6}$ which is (conformally) Calabi-Yau. 
For SU(3) structure manifolds, the supersymmetry conditions for the forms $\alpha$ and $w$ can be simplified using the formulae discussed in appendix A, in particular equation (A.6). With simple manipulations we can write the set of constraints (2.35)-(2.38) as

$$
\begin{aligned}
\eta_{+}^{\dagger} w \eta_{+} & =\eta_{+}^{\dagger} \alpha \eta_{+}=0 \\
\operatorname{Re}\left[e^{i \theta} \eta_{+}^{\dagger} \gamma^{m n}\left(e^{-\phi-i \theta} \alpha-i w\right) \eta_{+}\right] & =\operatorname{Re}\left[e^{i \theta} \eta_{+}^{\dagger}\left(e^{-\phi-i \theta} \alpha+i w\right) \gamma^{m n} \eta_{+}\right]=0, \\
\eta_{-}^{\dagger} \gamma^{n}\left(e^{-\phi-i \theta} \alpha-i w\right) \eta_{+} & =\eta_{-}^{\dagger}\left(e^{-\phi+i \theta} \alpha-i w\right) \gamma^{n} \eta_{+}=0
\end{aligned}
$$

or, equivalently,

$$
\begin{aligned}
\left(\bar{\Phi}_{+}, \alpha\right)_{6} & =\left(\bar{\Phi}_{+}, w\right)_{6}=0, \\
\operatorname{Im}\left[\left(\bar{\Phi}_{+}, \gamma^{m n}\left(e^{-\phi-i \theta} \alpha-i w\right)\right)_{6}\right] & =\operatorname{Im}\left[\left(\bar{\Phi}_{+},\left(e^{-\phi-i \theta} \alpha+i w\right) \gamma^{m n}\right)_{6}\right]=0, \\
\left(\Phi_{-}, \gamma^{n}\left(e^{-\phi-i \theta} \alpha-i w\right)\right)_{6} & =\left(\Phi_{-},\left(e^{-\phi+i \theta} \alpha-i w\right) \gamma^{n}\right)_{6}=0 .
\end{aligned}
$$

Before discussing explicit solutions, let us analyze the content of the supersymmetry conditions (4.7)-(4.9). Consider first solutions where the polyform $w$ only has a two-form component $w_{2}$. Then the general solution of the supersymmetry conditions is obtained by requiring that $\alpha$ and $w_{2}$ are $(1,1)$ and primitive. ${ }^{7}$ The requirement that $\alpha$ and $w_{2}$ are primitive is just equation (4.4), or, equivalently, (4.7). If $\alpha$ and $w_{2}$ are also $(1,1)$ all other equations are automatically satisfied since any real two-form, $C_{2},(1,1)$ and primitive satisfies

$$
C_{2} \eta_{+}=\eta_{ \pm}^{\dagger} C_{2}=0
$$

In the special case $\theta=0$, a $(2,0)$ component in $\left(e^{-\phi} \alpha-i w_{2}\right)$ will also be allowed. In fact, in this case

$$
\left(e^{-\phi} \alpha-i w_{2}\right) \eta_{+}=\eta_{-}^{\dagger}\left(e^{-\phi} \alpha-i w_{2}\right)=\eta_{+}^{\dagger}\left(e^{-\phi} \alpha+i w_{2}\right)=0 .
$$

In the general case, $\alpha$ is still required to be primitive. The conditions on the other forms are more involved. Let us notice that all conditions can be satisfied at once if

$$
\alpha \eta_{+}=w \eta_{+}=\lambda(w) \eta_{+}=0,
$$

since then $\eta_{ \pm} \alpha=\eta_{ \pm} w=0 .{ }^{8}$

In type IIB, the modifications (3.11) one has to make to the supersymmetry conditions when the spinor depends on $\varphi$ greatly simplify for $\theta=0$, and become equivalent to a shift in the zero form component of $w: w_{0} \rightarrow w_{0}-2 k e^{-\phi}$.

\subsection{Examples}

Supersymmetric Lif $_{4}$ solutions based on $A d S_{5}$ vacua have been discussed in [5, 6, 10, 18]. As we will discuss now, solutions of this kind can be easily cast in our formalism. In the same spirit, we will show how flows among $A d S_{5}$ vacua can give rise to flows among the corresponding $\mathrm{Lif}_{4}$ vacua.

\footnotetext{
${ }^{7} \mathrm{~A}$ two-form $C$ is primitive if its contraction with $J$ is zero, $C_{m n} J^{m n}=\eta_{+}^{\dagger} C \eta_{+}=0$; this is equivalent to $\left(\bar{\Phi}_{+}, C\right)=C \wedge J^{2}=0$.

${ }^{8}$ For a generic even polyform $A, A \eta_{+}=0$ implies $\eta_{+}^{\dagger} \lambda(\bar{A})=\eta_{-}^{\dagger} \lambda(A)=0$.
} 


\subsection{1 $\mathrm{Lif}_{4}$ from $A d S_{5}$ solutions}

A first example of solutions fitting the ansatz of sections 2 and 3 is the family found in [6], which we review here. They represent type IIA and IIB Lif 4 backgrounds where the internal manifold is a U(1) fibration over a five-dimensional Sasaki-Einstein manifold $Y$. Both IIA and IIB solutions are based on the standard type IIB vacuum of the form $A d S_{5} \times Y$

$$
\mathrm{d} s_{10}^{2}=r^{2}\left(\eta_{\mu \nu} \mathrm{d} x^{\mu} \mathrm{d} x^{\nu}\right)^{2}+\frac{1}{r^{2}}\left(\mathrm{~d} r^{2}+r^{2} \mathrm{~d} s_{Y}^{2}\right),
$$

where the internal manifold $M_{6}$ is a conformal Calabi-Yau cone over the Sasaki-Einstein $Y$. The dilaton $\phi$ is constant and set to zero, and, in the notations of sections 2 and 3, there is only a five-form flux

$$
h=0, \quad * f=4 \frac{\mathrm{d} r}{r} .
$$

The pure spinors are given by equation (4.3) with $\theta=0$ and

$$
J=\frac{1}{r^{2}} J_{\mathrm{CY}} \quad \Omega=\frac{1}{r^{2}} \Omega_{\mathrm{CY}} .
$$

The supersymmetry conditions (2.44)-(2.46) are trivially satisfied since $\mathrm{d} J_{C Y}=\mathrm{d} \Omega_{C Y}=0$. We normalized the spinors with $c_{+}=2$ (this choice implies $\left\|\eta_{+}\right\|^{2}=e^{A}$, cfr equation $(2.43))$.

Using (2.47) we can write the associated non-relativistic type IIA solution [6] as

$$
\mathrm{d} s_{10}^{2}=-\frac{r^{4}}{q^{2}} \mathrm{~d} t^{2}+r^{2}\left(\mathrm{~d} x^{2}+\mathrm{d} y^{2}\right)+\frac{\mathrm{d} r^{2}}{r^{2}}+\frac{1}{q^{2}}(\mathrm{~d} \varphi+\mu)^{2}+\mathrm{d} s_{Y}^{2},
$$

where $\mathrm{d} \mu=\alpha$. The dilaton is

$$
e^{-2 \phi}=q^{2} .
$$

The forms $\alpha$ and $w$ must satisfy equations (2.48)-(2.52). The simplest way to solve these constraints is to take only two-forms $\alpha$ and $w_{2}$ which are closed and co-closed on $Y$, or, equivalently harmonic on $Y$. These exist on all Sasaki-Einstein manifolds with $b_{2} \neq 0$. They can be primitive and of type $(1,1)$ so that, as discussed above, they preserve supersymmetry. The fluxes then have the form $[6]$

$$
\begin{aligned}
B & =\frac{r^{2}}{q^{2}} \mathrm{~d} t \wedge(\mathrm{d} \varphi+\mu), \\
F_{2} & =w_{2}, \\
F_{4} & =-4 r^{3} \mathrm{~d} t \wedge \mathrm{d} x^{1} \wedge \mathrm{d} x^{2} \wedge \mathrm{d} r+\frac{r^{2}}{q^{2}} \mathrm{~d} t \wedge(\mathrm{d} \varphi+\mu) \wedge w_{2} .
\end{aligned}
$$

The function $q$ on $Y$ must satisfy (2.57)

$$
4 q^{2}-\square_{Y} q^{2}=|\alpha|^{2}+\left|w_{2}\right|^{2} .
$$

Explicit solutions of this equation have been found in [6] in the case of $T^{1,1}$ and some $Y^{p, q}$. For $T^{1,1}$ the function $q$ is constant. Obviously we cannot find solutions of this type on $S^{5}$ since there are no harmonic forms on $S^{5}$. 
It is interesting to look at the form of the supersymmetry parameters. They satisfy conditions (2.17) and (2.18). For the solutions with $\eta_{+}^{1}=\eta_{+}^{2}$ we see from equation (2.19) that

$$
\Gamma^{0 x y} \epsilon_{1}=\epsilon_{2}
$$

where we took into account the redefinition $\hat{\eta}_{1} \rightarrow \hat{\gamma}^{x y} \hat{\eta}_{1}$. This condition is reminiscent of the original D3 brane condition.

The case of type IIB is analogous. The metric is now of the form (3.1)

$$
\mathrm{d} s_{10}^{2}=-2 r^{2} \mathrm{~d} t \mathrm{~d} \varphi+r^{2}\left(\mathrm{~d} x^{2}+\mathrm{d} y^{2}\right)+\frac{\mathrm{d} r^{2}}{r^{2}}+q^{2} \mathrm{~d} \varphi^{2}+\mathrm{d} s_{Y}^{2}
$$

and the forms $\alpha$ and $w$ enter in the NS-NS and RR-RR fluxes as in (3.4) and (3.5). Notice that the fluxes are proportional to $\mathrm{d} \varphi$ and the time-translation Killing vector is null. We still have solutions based on harmonic two-forms on $Y$ [6].

In type IIB we can also have solutions with an explicit dependence on $\varphi$. These have been discussed in details in $[5,6,10,18,19]$. We just consider the simplest case. Set all the forms $\alpha$ and $w$ equal to zero, except for $w_{0}$. The resulting solution then can exist also on $S^{5}$. A non-zero $w_{0}$ corresponds to a one-form flux

$$
F_{1}=w_{0} \mathrm{~d} \varphi
$$

or, equivalently, to a linear profile for the axion $C_{0}=w_{0} \varphi$. As discussed at the beginning of this section, with a $\varphi$ dependent spinor we can compensate the non-zero value of $w_{0}$ (since $\theta=0$ ) and have a supersymmetric solution. The equation (2.57) for $q$ reads

$$
4 q^{2}-\square_{Y} q^{2}=\left|w_{0}\right|^{2}
$$

and can be solved with a constant function $q$. When $\varphi$ is compact, some quantization condition should be imposed on the parameters in order to have a consistent solution [18]. The supersymmetric solution with non-compact $\varphi$ has been interpreted in [19] as describing a deformation of $\mathcal{N}=4 \mathrm{SYM}$ (or, more generally, of the four-dimensional superconformal theory associated with $Y$ ) in the presence of a linear theta angle.

\subsubsection{Asymptotically Lif $_{4}$ solutions}

The ansatz described in sections 2 and 3 allows to show in an elegant way that many supersymmetric asymptotically $A d S_{5}$ backgrounds in type IIB descend to analogous solutions with asymptotic $L_{i f}$ vacua. In particular, starting from supersymmetric domain walls in type IIB we expect to find solutions interpolating between Lif $_{4}$ vacua. In the gauge/gravity correspondence, a domain-wall connecting two $A d S_{5}$ vacua is interpreted as a RG flow between the corresponding $C F T_{4} \mathrm{~S}[20,21]$. The interpolation between $\mathrm{Lif}_{4}$ vacua has obviously an analogous interpretation in terms of RG flows between $\mathrm{NRCFT}_{3}$.

As an example, we consider the case of $4 \mathrm{~d}$ RG flows with $\mathrm{SU}(3)$ structure based on internal manifolds that are (non-conical) $C Y_{6}$

$$
\mathrm{d} s_{10}^{2}=e^{2 A}\left(\eta_{\mu \nu} \mathrm{d} x^{\mu} \mathrm{d} x^{\nu}\right)+e^{-2 A} \mathrm{~d} s_{C Y_{6}}^{2}, \quad \mu=0, . ., 3 .
$$


The dilaton is constant and set to zero, and all fluxes are zero except for the five-form

$$
* f=4 \mathrm{~d} A,
$$

where $e^{-4 A}$ is required to be a harmonic function on $C Y_{6}$. The pure spinors are given again by equation (4.3) with $\theta=0$, and

$$
J=e^{-2 A} J_{\mathrm{CY}} \quad \Omega=e^{-2 A} \Omega_{\mathrm{CY}} .
$$

The first regular domain-wall which is conformally Calabi-Yau was found in [22] and interpolates between $A d S_{5} \times T^{1,1}$ and $A d S_{5} \times S^{5}$. It is obtained by displacing a large number of D3 branes on the resolved conifold. Near the position of the D3 branes a throat will emerge recreating $A d S_{5} \times S^{5}$. The solution has a natural field theory interpretation in terms of baryonic VEVs. By moving in the baryonic moduli space of the conifold theory we can flow in the IR to pure $N=4 \mathrm{SYM}$.

Similar solutions exist for all resolved $C Y_{6}$ [23]. In fact, it is well known that the web of four-dimensional quiver gauge theories associated to D3 branes sitting at conical CalabiYau singularities can be connected by RG flows induced by baryonic operators. On the geometrical side, the corresponding $C Y_{6}$ are obtained by resolutions. When the resolution of the cone $C\left(Y_{1}\right)$ is only partial and the $C Y_{6}$ has still local conical singularities of the form $C\left(Y_{2}\right)$ we can engineer a domain wall interpolating between $A d S_{5} \times Y_{1}$ and $A d S_{5} \times Y_{2}$ by putting D3 branes at the local singularity on the resolved Calabi-Yau.

From each of these solutions, we have type IIB domain-walls interpolating between the Lifshitz solutions corresponding to $Y_{1}$ and $Y_{2}$

$$
\mathrm{d} s_{10}^{2}=-2 e^{2 A} \mathrm{~d} t \mathrm{~d} \varphi+e^{2 A}\left(\mathrm{~d} x^{2}+\mathrm{d} y^{2}\right)+q^{2} \mathrm{~d} \varphi^{2}+e^{-2 A} \mathrm{~d} s_{C Y_{6}}^{2},
$$

with a linear axion

$$
F_{1}=w_{0} \mathrm{~d} \varphi
$$

The function $q$ is determined by

$$
-\square_{C Y_{6}}\left(q^{2} e^{-2 A}\right)=e^{-4 A}\left|w_{0}\right|^{2},
$$

with $q$ becoming constant in the asymptotic regions.

More generally, we can have type IIA and type IIB solutions with two-forms $\alpha$ and $w_{2}$. The type IIB metric would be still of the form (4.29) and in the type IIA case we have

$$
\mathrm{d} s_{10}^{2}=-e^{4 A} \mathrm{~d} t^{2}+e^{2 A}\left(\mathrm{~d} x^{2}+\mathrm{d} y^{2}\right)+\left(e^{1}\right)^{2}+e^{-2 A} \mathrm{~d} s_{C Y_{6}}^{2} .
$$

From equations (2.48)-(2.52) we see that the two-forms $\alpha$ and $w_{2}$ must be closed and co-closed on the $C Y_{6}$

$$
\mathrm{d} \alpha=\mathrm{d} w_{2}=0 \quad \mathrm{~d}\left(*_{C Y_{6}} \alpha\right)=\mathrm{d}\left(*_{C Y_{6}} w_{2}\right)=0 .
$$

Such harmonic forms exist on resolved $C Y_{6}$ with $b_{2} \neq 0$. Explicit solutions for the resolved conifold and its quotients can be found in [24, 25] and a general discussion in [23]. In this case $q$ is determined by

$$
-\square_{C Y_{6}}\left(q^{2} e^{-2 A}\right)=e^{-4 A}\left(|\alpha|^{2}+\left|w_{2}\right|^{2}\right) .
$$


In the original example [22] the solution of this equation becomes singular in the IR, since $S^{5}$ has no harmonic two-forms. For more general solutions interpolating between different Sasaki-Einstein manifolds we expect the existence of regular solutions.

The field theory realization of the $N_{R C F T_{3}}$ dual to the existing Lif $_{4}$ vacua is still unclear. We may expect to understand them in terms of Chern-Simons gauge theories (see [19] for an attempt). The existence of a map between (many) $\mathrm{CFT}_{4} \mathrm{~s}$ to $\mathrm{NRCFT}_{3} \mathrm{~s}$ and the corresponding flows suggests that it should be possible to understand and classify these $N R C F T_{3}$ in terms of the better known parent four-dimensional quiver gauge theories. For this reason, it would be interesting to perform a full scan of the Sasaki-Einstein manifolds that give rise to $\mathrm{Lif}_{4}$ vacua and of the allowed (regular) flows between them. Moreover, as recently pointed out [26], three-dimensional theories with $z=2$ have a trace anomaly which gives rise to a single central charge in models with a holographic $L i f_{4}$ dual. This central charge is expected to decrease along a holographic flow on general grounds [20, 21] and it would be very interesting to evaluate it for the models at hand.

\subsubsection{Solutions with hyperscaling violation}

The $\mathrm{Lif}_{4}$ examples we have considered in section 4.1.1 are scale-invariant; the dilaton and all other scalar functions are required to be independent of the radial coordinate $r$. If we allow the dilaton, or other quantities, to have a non-trivial profile in $r$ we can realize more general solutions with a dynamical critical exponent $z$ and a hyperscaling violation exponent $\theta$ corresponding to the class of metrics $[27,28]$

$$
\mathrm{d} s^{2}=u^{-2\left(1-\frac{\theta}{D}\right)}\left(-u^{-2(z-1)} \mathrm{d} t^{2}+\sum_{i=1}^{D}\left(\mathrm{~d} x^{i}\right)^{2}+\mathrm{d} u^{2}\right),
$$

with $u=1 / r$. The metric is conformal to the Lifschitz space-time but transforms as $\mathrm{d} s^{2} \rightarrow \lambda^{2 \theta / D} \mathrm{~d} s^{2}$ under the rescaling

$$
t \rightarrow \lambda^{z} t \quad x^{i} \rightarrow \lambda x^{i} \quad u \rightarrow \lambda u .
$$

Obviously these solutions have singularities for small or large $r$, and can be only considered as an effective description of the physics in some range of the radial coordinate. For large and small $r$ we may expect the solution to have a different form, corresponding to an AdS or Lifshitz vacuum, or to a more general regular solution. As pointed out in [28] a very simple physical realization of such system with $z=1$ and $\theta=-\frac{1}{3}$ is given by D2 branes for a given range of values of $r$.

Starting with a general IIB solution of the form (4.26) with constant dilaton we can obtain a non-relativistic solution with running dilaton if $q$ has an $r$-dependence (see (2.47) and (3.9)). The function $q$ satisfies equation (2.57) which now reads

$$
-\square_{C Y_{6}}\left(q^{2} e^{-2 A}\right)=e^{-4 A}\left(|\alpha|^{2}+|w|^{2}\right) .
$$

The general solution of this equation is obtained from a particular one by adding the solution of the homogeneous equation $\square_{C Y_{6}}\left(q^{2} e^{-2 A}\right)=0$. 
Let us consider, for instance, the case of conic Calabi-Yau manifolds of section 4.1.1, with $e^{A}=r$. With zero internal forms $\alpha=w=0$ and $q=e^{-A}=1 / r$, we obtain the non-relativistic type IIA solution

$$
\mathrm{d} s_{10}^{2}=-r^{6} \mathrm{~d} t^{2}+r^{2}\left(\mathrm{~d} x^{2}+\mathrm{d} y^{2}\right)+r^{2} d \varphi^{2}+\frac{\mathrm{d} r^{2}}{r^{2}}+\mathrm{d} s_{Y}^{2},
$$

with $e^{\phi_{A}}=r$ and

$$
H^{I I A}=\mathrm{d}\left(r^{4} \mathrm{~d} t \wedge \mathrm{d} \varphi\right), \quad F_{4}=-4 r^{3} \mathrm{~d} t \wedge \mathrm{d} x^{1} \wedge \mathrm{d} x^{2} \wedge \mathrm{d} r .
$$

There is also an analogous type IIB solution. Solutions of this kind, with $z=3$ and $\theta=2,{ }^{9}$ have been discussed in $[29,30]$. The solution only has a limited range of validity. For example, for large $r$ the dilaton grows and invalidates the solution. We can have a different UV completion if we use the more general solution

$$
q^{2}=c_{1} r^{2}+c_{2} / r^{2}
$$

In this case we obtain a metric that for large $r$ is a sort of T-dual of the $A d S_{5} \times Y$ type IIB solution and reduces in the IR to the metric with hyperscaling violation.

If we further add internal two- forms $\alpha$ and $w_{2}$ on $Y$ we can have more general solutions with

$$
q^{2}=c_{0}+c_{1} r^{2}+c_{2} / r^{2}
$$

where $c_{0}$ is a radial independent solution of (4.21). For $c_{2}=0$ these solutions would interpolate between a T-dual of $A d S_{5} \times Y$ in the UV and the Lif $_{4}$ solution discussed in section 4.1.1 in the IR.

\section{Conclusions}

We have discussed a general framework to determine supersymmetric type II nonrelativistic solutions with exact or asymptotic scale invariance. As already emerged from previous investigations $[6,10]$, there is a clear correspondence between anisotropic $d$-dimensional vacua and relativistic solutions in $d+1$ dimensions. The known supersymmetric Lif $_{4}$ solutions have $z=2$ and descend from $A d S_{5}$ vacua. This correspondence between $A d S_{d+1}$ and $L i f_{d}$ vacua is certainly intriguing and deserve further study. In particular it may be useful in explicitly constructing the three-dimensional theories dual to Lifshitz vacua.

Beside clarifying the correspondence between $d+1$ and $d$-dimensional vacua, our formalism can be applied to the search of new solutions. In this paper we have only considered the simplest generalizations of the solutions found in [6], based on $C Y_{6}$ four-dimensional vacua. There are other obvious directions of investigation. For simplicity, we have only considered SU(3) structures. The case of SU(2) structure is considerably more involved.

\footnotetext{
${ }^{9}$ The hyperscaling violation refers to the scaling of the effective $D+2=5$ dimensional metric in Einstein frame.
} 
However, many interesting $A d S_{5}$ solutions with three-form fluxes, including the PilchWarner solution (PW) [31] and the beta-deformation of Sasaki-Einstein backgrounds [32], have $\mathrm{SU}(2)$ structure $[33,34]$ and we expect the existence of corresponding $\mathrm{Lif}_{4}$ solutions.

The formalism could be applied also to the study of confining solutions. It would be quite interesting to see if known relativistic four-dimensional confining solutions descend to non-relativistic solutions with asymptotically Lifshitz scaling and a confining behaviour in the IR. In the case of the obvious candidates (the Klebanov-Strassler [35], the Maldacena-Nunez [36] and the interpolating baryonic branch [37] solutions) it is not immediately obvious how to find a set of polyforms $(\alpha, w)$ satisfying all constraints (2.48)(2.52) and (2.53)-(2.56) and maintaining regularity. However, it is not excluded that a generalization of this construction will give interesting solutions. We leave the detailed analysis of these and similar cases to future work.

\section{Acknowledgments}

We wish to thank A. Tomasiello for interesting discussions. M. P. is partially supported by the Institut de Physique Théorique, du CEA. A. Z. is partially supported by INFN and the MIUR-PRIN contract 2009-KHZKRX.

\section{A Notations and useful formulae}

We use the notation of [15] to which we refer for more details. To a differential form we can associate a bispinor via the Clifford map

$$
C_{k} \equiv \frac{1}{k !} C_{M_{1} \ldots M_{k}} d x^{M_{1}} \wedge \ldots \wedge d x^{M_{k}} \longrightarrow \ell_{k} \equiv \frac{1}{k !} C_{M_{1} \ldots M_{k}} \gamma^{M_{1} \ldots M_{k}}
$$

The Clifford product is

$$
\gamma^{M} C_{k}=\left(d x^{M} \wedge+\iota^{M}\right) C_{k}, \quad C_{k} \gamma^{M}=(-)^{k}\left(d x^{M} \wedge-\iota^{M}\right) C_{k},
$$

where $\iota^{M} \equiv g^{M N} \iota_{N} \equiv g^{M N} \iota_{\partial / \partial x^{N}}$. In this formulae and in the main text the "slash" symbol is usually understood. We keep only when needed to clarify the origin of few signs. We take hermitian (imaginary and antisymmetric) six-dimensional gamma matrices. The six-dimensional chirality is defined through

$$
\gamma_{7}=-i \gamma^{1} \cdots \gamma^{6}
$$

which on bispinors gives

$$
\gamma_{7} \ell^{\prime}=-i * \lambda\left(\ell^{\prime}\right)
$$

where $\lambda\left(C_{p}\right)=(-1)^{[p / 2]} C_{p}$, if $C_{p}$ is a form of degree $p$. This equation is consistent with our definition of the star product

$$
* C \wedge C=|C|^{2} \mathrm{vol}
$$


The six-dimensional Mukai pairing reads

$$
(A, B)_{6}=(A \wedge \lambda B)_{6}=-\frac{i}{8}(-1)^{\operatorname{deg} A} \operatorname{Tr}\left(\gamma_{7}(\bar{A})^{\dagger} \not B\right) .
$$

In using this equation, we must remember that the gamma matrices are imaginary so that one must be careful with signs when converting the conjugate odd forms into bispinor. The only relevant case for us comes with the complex conjugate of $\Phi_{-}$

$$
\left(\bar{\Phi}_{-}\right)=-\overline{\left(\Phi_{-}\right)} \text {. }
$$

The pure spinors

$$
\Phi_{+}=\eta_{+}^{1} \eta_{+}^{2 \dagger}, \quad \Phi_{-}=\eta_{+}^{1} \eta_{-}^{2 \dagger},
$$

satisfy the identities

$$
\left(\bar{\Phi}_{+}, \Phi_{+}\right)_{6}=\left(\bar{\Phi}_{-}, \Phi_{-}\right)_{6}, \quad\left(\Phi_{+}, Z \Phi_{-}\right)_{6}=\left(\Phi_{+}, Z \bar{\Phi}_{-}\right)_{6}=0, \quad Z \in T \oplus T^{*},
$$

Using (A.6) we can also derive the following identities

$$
\begin{aligned}
& \left(\Phi_{+}, X \Phi_{-} Y\right)_{6}=-(-1)^{\left[\frac{\operatorname{deg} Y}{2}\right]}\left(\Phi_{-}, X \Phi_{+} Y\right)_{6}=\frac{i}{8}\left(\eta_{-}^{1 \dagger} X \eta_{+}^{1}\right)\left(\eta_{-}^{2 \dagger} Y \eta_{-}^{2}\right) \\
& \left(\bar{\Phi}_{+}, X \Phi_{+} Y\right)_{6}=(-1)^{\left[\frac{\operatorname{deg} Y}{2}\right]}\left(\bar{\Phi}_{-}, X \Phi_{-} Y\right)_{6}=-\frac{i}{8}\left(\eta_{+}^{1 \dagger} X \eta_{+}^{1}\right)\left(\eta_{+}^{2 \dagger} Y \eta_{+}^{2}\right), \\
& \left(\Phi_{+}, X \bar{\Phi}_{-} Y\right)_{6}=(-1)^{\left[\frac{\operatorname{deg} X}{2}\right]}\left(\bar{\Phi}_{-}, X \Phi_{+} Y\right)_{6}=-\frac{i}{8}\left(\eta_{-}^{1 \dagger} X \eta_{-}^{1}\right)\left(\eta_{+}^{2 \dagger} Y \eta_{-}^{2}\right),
\end{aligned}
$$

where $X$ and $Y$ are generic products of gamma matrices of the form $\gamma^{\left\{\mu_{1} \cdots \mu_{k}\right\}}$ acting in the Mukai pairing via Clifford multiplication (A.2). It can be useful to remember that $Y^{T}=(-1)^{\left[\frac{\operatorname{deg} Y}{2}\right]} Y$ and, as obvious from $(4.3), \eta_{+}^{i \dagger} X \eta_{+}^{i}$ is non zero only for even $X$ and $\eta_{-}^{i \dagger} X \eta_{+}^{i}$ only for odd $X$. Other useful identities which follow from (A.4) are

$$
\begin{aligned}
\left(\bar{\Phi}_{ \pm}, \gamma^{m} * \lambda(f)\right)_{6} & =\left(\bar{\Phi}_{ \pm}, \gamma^{m}(-i f)\right)_{6} \\
\left(\bar{\Phi}_{ \pm}, * \lambda(f) \gamma^{m}\right)_{6} & =\left(\bar{\Phi}_{ \pm}, i f \gamma^{m}\right)_{6}
\end{aligned}
$$

\section{B The conditions for supersymmetry}

In this appendix we show how to simplify conditions (1.12) and (1.13). We will do it for the case of type IIA, type IIB being completely analogous. We start from $K_{1}$ and $K_{2}$ given in $(2.23)$

$$
K_{1}=\frac{C}{16}\left\|\eta_{+}\right\|^{2}\left(e^{0}+e^{1}\right), \quad K_{2}=\frac{C}{16}\left\|\eta_{+}\right\|^{2}\left(e^{0}-e^{1}\right) .
$$

Setting $K_{i}=e_{-i}$, we can define two basis of vielbeine as $\left(e_{-i}, e_{+i}, e_{I}\right)$, with

$$
e_{+1}=\frac{1}{4} e^{-A_{1}}\left(e^{1}-e^{0}\right) \quad e_{+2}=-\frac{1}{4} e^{-A_{1}}\left(e^{0}+e^{1}\right) .
$$

where we use the normalization of (1.9). 
Using an identity analogous to (A.6) (see also formula (B.33) in [15])

$$
\left(e_{+1} \cdot \Phi \cdot e_{+2}, C\right)=\frac{1}{32}(-1)^{\operatorname{deg} \Phi} \bar{\epsilon}_{1} e_{+1} C e_{+2} \epsilon_{2},
$$

where $C$ is a generic bispinor, it is easy to verify that

$$
\left(e_{+1} \cdot \Phi \cdot e_{+2}, C \cdot e_{+2}\right)=\left(e_{+1} \cdot \Phi \cdot e_{+2}, e_{+1} \cdot C\right)=0
$$

and

$$
\left(e_{+1} \cdot \Phi \cdot e_{+2}, \Gamma_{10} C\right)=\left(e_{+1} \cdot \Phi \cdot e_{+2}, C\right) .
$$

The first identity follows from the vanishing of the square of the null vector $e_{+i}$ and the second from the fact that $\epsilon_{1}$ has positive chirality.

Let us now consider (1.12). The left-hand side of the Mukai paring gives

$$
e_{+1} \cdot \Phi \cdot e_{+2}=\frac{1}{8} C e^{-2 A_{1}}\left(1-e^{01}\right) \Phi_{8}
$$

The $\mathrm{d}_{H}$ terms can be written as

$$
\begin{aligned}
\mathrm{d}_{H}\left(e^{-\phi_{A}} \Phi \cdot e_{+2}\right)= & \mathrm{d}_{H}\left(e^{-\phi_{A}-A_{1}} \frac{C}{4} \Phi_{8}\left(e^{0}+e^{1}\right)\right) \\
= & \mathrm{d}_{h}\left(e^{-\phi_{A}-A_{1}} \frac{C}{4} \Phi_{8}\right) \cdot\left(e^{0}+e^{1}\right)+e^{-\phi_{A}-A_{1}} \frac{C}{4} \Phi_{8} \mathrm{~d}\left(e^{0}+e^{1}\right) \\
& -\mathrm{d}\left(e^{01}\right) \wedge e^{-\phi_{A}-A_{1}} \frac{C}{4} \Phi_{8}\left(e^{0}+e^{1}\right) \\
= & \mathrm{d}_{h}\left(e^{-\phi_{A}-A_{1}} \frac{C}{4} \Phi_{8}\right) \cdot\left(e^{0}+e^{1}\right) \\
& +e^{-\phi_{A}-A_{1}} \frac{C}{4} \Phi_{8}\left(\mathrm{~d} A_{1} e^{0}-\frac{\mathrm{d} q}{q} e^{1}+\frac{\alpha}{q}\left(1+e^{01}\right)\right)
\end{aligned}
$$

while, from (B.2) it follows immediately that

$$
\mathrm{d}^{\dagger}\left(e^{-2 \phi_{A}} e_{+2}\right)=0
$$

Finally, the fluxes can be written as

$$
F^{I I A}=\left(1+\Gamma_{10}\right)\left[-q e^{1} f+\left(1+e^{01}\right) w\right],
$$

and, when inserted in (1.12), we can use (B.5) to replace $\Gamma_{10}$ in (B.9) with the identity matrix. Notice also that the first term on the right hand side of equation (B.7) does not contribute to (1.12) because of the identities (B.4). Similarly, we can also manipulate the remaining terms dropping all pieces of the form ()$\cdot\left(e^{0}+e^{1}\right)$. We finally obtain

$$
\left(\left(1-e^{01}\right) \Phi_{8}, \Gamma^{M N}\left[\left(e^{-\phi_{A}-A_{1}} \frac{C}{8}\left(\mathrm{~d} A_{1}+\frac{\mathrm{d} q}{q}\right) \Phi_{8}+q f\right)\left(e^{0}-e^{1}\right)+\left(e^{-\phi_{A}-A_{1}} \frac{C}{4 q} \alpha \Phi_{8}-2 w\right)\left(1+e^{01}\right)\right]\right) \text {. }
$$

The ten-dimensional Mukai pairing (B.10) can be reduced to a six-dimensional one using the explicit form of $\Phi_{8}$ given in equation (2.28). We need to distinguish various cases for $M$ and $N$. 
When $M=0$ or $M=5$ the only non-zero contributions come from the term proportional to $e^{0}-e^{1}$ on the right hand side. Not unexpectedly, these contributions are similar to those found in the analysis of four-dimensional vacua in [15]. When $N=x, y$ most of the terms vanish due to (A.9) and we obtain the constraint

$$
\left(\Phi_{-}, f\right)_{6}=0
$$

while $N=n$ gives

$$
\operatorname{Im}\left[\left(\bar{\Phi}_{+}, \gamma^{n}\left[e^{-A_{1}-\phi_{A}} \frac{C}{4}\left(\mathrm{~d} A_{1}+\frac{\mathrm{d} q}{q}\right) \Phi_{+}-q * \lambda f\right]\right)\right]=0
$$

where we used (A.10) and (A.11). These two conditions are actually implies by the supersymmetry equations. Indeed, using (A.11) and (2.31), (B.11) can be written as

$$
\left(\Phi_{-}, * f\right)_{6} \sim\left(\Phi_{-}, \mathrm{d}_{h} \Phi_{+}\right)_{6}=\left(\mathrm{d}_{h} \Phi_{-}, \Phi_{+}\right)_{6}=0
$$

due to (2.32) and (A.9). Since (2.30) and (2.31) can be combined in

$$
\mathrm{d}_{h}\left(\frac{C}{2} e^{A_{2}-\phi_{A}} \bar{\Phi}_{+}\right)=-\frac{C}{2} e^{A_{2}-\phi_{A}} \mathrm{~d} A_{2} \Phi_{+}+q e^{A_{1}+A_{2}} * \lambda f
$$

we see that also (B.12) can be written as

$$
\operatorname{Im}\left[\left(\bar{\Phi}_{+}, \gamma^{n} \mathrm{~d}_{h}\left(\frac{C}{2} e^{A_{2}-\phi_{A}} \bar{\Phi}_{+}\right)\right)_{6}\right]=0
$$

provided we set $e^{A_{1}}=e^{2 A_{2}} / q$. This equation is automatically satisfied since $\Phi_{+}$is a pure spinor. Indeed,

$$
\left(\bar{\Phi}_{+}, X \bar{\Phi}_{+}\right)
$$

can be different from zero only for an insertion $X$ of six gamma matrices. On the contrary $d_{h}$ can bring a maximum of three gamma since it is odd and $d$ can only change the complex type $(p, q)$ of a form by a maximum of two units in $p$ and $q$.

For all other values of $M$ and $N$, (B.10) gets contributions only from the term proportional to $\left(1+e^{01}\right) . M=0$ and $N=5$ and $M=x, N=y$ give

$$
\left(\bar{\Phi}_{+}, \frac{C}{16 q} e^{-A_{1}-\phi_{A}} \alpha \cdot \Phi_{+}-i w\right)_{6}=0
$$

where $\cdot$ denote the Clifford product, while for $M=m$ and $N=n$ we obtain

$$
\operatorname{Im}\left[\left(\bar{\Phi}_{+}, \gamma^{m n}\left(\frac{C}{16 q} e^{-A_{1}-\phi_{A}} \alpha \cdot \Phi_{+}-i w\right)\right)_{6}\right]=0 .
$$

Finally $M=x, y$, and $N=n$ give

$$
\left(\Phi_{-}, \gamma^{n}\left(\frac{C}{4 q} e^{-A_{1}-\phi_{A}} \alpha \wedge \Phi_{+}-i w\right)\right)_{6}=0
$$


In deriving the above conditions, we used repeatedly the identities (A.10). Using (2.25) we recover half of the conditions (2.35)-(2.38). Equation (1.13) can be treated in a similar way and gives the remaining half of the conditions.

The computation in type IIB is similar and it will not be reported here. Let us simply note that, with spinors depending on $\varphi$ as in (3.10), there is an extra contribution from $\mathrm{d}_{H}\left(e^{-\phi_{A}} \Phi \cdot e_{+2}\right)$ coming from the component of $\mathrm{d} \Phi_{8}$ along $e^{-}$; this term is responsible for the replacement (3.11).

Open Access. This article is distributed under the terms of the Creative Commons Attribution License which permits any use, distribution and reproduction in any medium, provided the original author(s) and source are credited.

\section{References}

[1] S. Kachru, X. Liu and M. Mulligan, Gravity duals of Lifshitz-like fixed points, Phys. Rev. D 78 (2008) 106005 [arXiv:0808.1725] [InSPIRE].

[2] M. Taylor, Non-relativistic holography, arXiv:0812.0530 [INSPIRE].

[3] W. Li, T. Nishioka and T. Takayanagi, Some no-go theorems for string duals of non-relativistic Lifshitz-like theories, JHEP 10 (2009) 015 [arXiv:0908. 0363] [INSPIRE].

[4] J. Blaaback, U.H. Danielsson and T. Van Riet, Lifshitz backgrounds from $10 D$ supergravity, JHEP 02 (2010) 095 [arXiv: 1001.4945] [INSPIRE].

[5] K. Balasubramanian and K. Narayan, Lifshitz spacetimes from AdS null and cosmological solutions, JHEP 08 (2010) 014 [arXiv: 1005.3291] [INSPIRE].

[6] A. Donos and J.P. Gauntlett, Lifshitz solutions of $D=10$ and $D=11$ supergravity, JHEP 12 (2010) 002 [arXiv: 1008.2062] [INSPIRE].

[7] A. Donos, J.P. Gauntlett, N. Kim and O. Varela, Wrapped M5-branes, consistent truncations and AdS/CMT, JHEP 12 (2010) 003 [arXiv: 1009.3805] [INSPIRE].

[8] R. Gregory, S.L. Parameswaran, G. Tasinato and I. Zavala, Lifshitz solutions in supergravity and string theory, JHEP 12 (2010) 047 [arXiv: 1009.3445] [INSPIRE].

[9] N. Halmagyi, M. Petrini and A. Zaffaroni, Non-relativistic solutions of $N=2$ gauged supergravity, JHEP 08 (2011) 041 [arXiv: 1102.5740] [INSPIRE].

[10] D. Cassani and A.F. Faedo, Constructing Lifshitz solutions from AdS, JHEP 05 (2011) 013 [arXiv: 1102.5344] [INSPIRE].

[11] N. Hitchin, Generalized Calabi-Yau manifolds, Quart. J. Math. Oxford Ser. 54 (2003) 281 [math/0209099] [INSPIRE].

[12] M. Gualtieri, Generalized complex geometry, math.DG/0401221 [INSPIRE].

[13] M. Graña, R. Minasian, M. Petrini and A. Tomasiello, Supersymmetric backgrounds from generalized Calabi-Yau manifolds, JHEP 08 (2004) 046 [hep-th/0406137] [INSPIRE].

[14] M. Graña, R. Minasian, M. Petrini and A. Tomasiello, Generalized structures of $N=1$ vacua, JHEP 11 (2005) 020 [hep-th/0505212] [INSPIRE].

[15] A. Tomasiello, Generalized structures of ten-dimensional supersymmetric solutions, JHEP 03 (2012) 073 [arXiv: 1109.2603] [INSPIRE]. 
[16] E. Bergshoeff, R. Kallosh, T. Ortín, D. Roest and A. Van Proeyen, New formulations of $D=10$ supersymmetry and D8-O8 domain walls, Class. Quant. Grav. 18 (2001) 3359 [hep-th/0103233] [INSPIRE].

[17] D. Lüst and D. Tsimpis, Supersymmetric AdS $S_{4}$ compactifications of IIA supergravity, JHEP 02 (2005) 027 [hep-th/0412250] [INSPIRE].

[18] W. Chemissany and J. Hartong, From D3-branes to Lifshitz space-times, Class. Quant. Grav. 28 (2011) 195011 [arXiv:1105.0612] [INSPIRE].

[19] K. Balasubramanian and J. McGreevy, String theory duals of Lifshitz-Chern-Simons gauge theories, arXiv:1111.0634 [INSPIRE].

[20] L. Girardello, M. Petrini, M. Porrati and A. Zaffaroni, Novel local CFT and exact results on perturbations of $N=4$ super Yang-Mills from AdS dynamics, JHEP 12 (1998) 022 [hep-th/9810126] [INSPIRE].

[21] D. Freedman, S. Gubser, K. Pilch and N. Warner, Renormalization group flows from holography supersymmetry and a c-theorem, Adv. Theor. Math. Phys. 3 (1999) 363 [hep-th/9904017] [INSPIRE].

[22] I.R. Klebanov and A. Murugan, Gauge/gravity duality and warped resolved conifold, JHEP 03 (2007) 042 [hep-th/0701064] [INSPIRE].

[23] D. Martelli and J. Sparks, Symmetry-breaking vacua and baryon condensates in AdS/CFT, Phys. Rev. D 79 (2009) 065009 [arXiv:0804.3999] [INSPIRE].

[24] H. Lü and J.F. Vazquez-Poritz, $S^{1}$ wrapped D3 branes on conifolds, Nucl. Phys. B 633 (2002) 114 [hep-th/0202175] [INSPIRE].

[25] I. Klebanov, A. Murugan, D. Rodriguez-Gomez and J. Ward, Goldstone bosons and global strings in a warped resolved conifold, JHEP 05 (2008) 090 [arXiv: 0712.2224] [INSPIRE].

[26] M. Baggio, J. de Boer and K. Holsheimer, Anomalous breaking of anisotropic scaling symmetry in the quantum Lifshitz model, arXiv:1112.6416 [INSPIRE].

[27] C. Charmousis, B. Gouteraux, B. Kim, E. Kiritsis and R. Meyer, Effective holographic theories for low-temperature condensed matter systems, JHEP 11 (2010) 151 [arXiv: 1005.4690] [INSPIRE].

[28] X. Dong, S. Harrison, S. Kachru, G. Torroba and H. Wang, Aspects of holography for theories with hyperscaling violation, JHEP 06 (2012) 041 [arXiv: 1201.1905] [INSPIRE].

[29] K. Narayan, On Lifshitz scaling and hyperscaling violation in string theory, Phys. Rev. D 85 (2012) 106006 [arXiv:1202.5935] [inSPIRE].

[30] P. Dey and S. Roy, Lifshitz-like space-time from intersecting branes in string/M theory, arXiv: 1203.5381 [INSPIRE].

[31] K. Pilch and N.P. Warner, A new supersymmetric compactification of chiral IIB supergravity, Phys. Lett. B 487 (2000) 22 [hep-th/0002192] [INSPIRE].

[32] O. Lunin and J.M. Maldacena, Deforming field theories with $\mathrm{U}(1) \times \mathrm{U}(1)$ global symmetry and their gravity duals, JHEP 05 (2005) 033 [hep-th/0502086] [INSPIRE].

[33] R. Minasian, M. Petrini and A. Zaffaroni, Gravity duals to deformed SYM theories and generalized complex geometry, JHEP 12 (2006) 055 [hep-th/0606257] [INSPIRE].

[34] A. Butti et al., On the geometry and the moduli space of beta-deformed quiver gauge theories, JHEP 07 (2008) 053 [arXiv: 0712.1215] [INSPIRE]. 
[35] I.R. Klebanov and M.J. Strassler, Supergravity and a confining gauge theory: duality cascades and $\chi S B$ resolution of naked singularities, JHEP 08 (2000) 052 [hep-th/0007191] [INSPIRE].

[36] J.M. Maldacena and C. Núñez, Towards the large- $N$ limit of pure $N=1$ super Yang-Mills, Phys. Rev. Lett. 86 (2001) 588 [hep-th/0008001] [INSPIRE].

[37] A. Butti, M. Graña, R. Minasian, M. Petrini and A. Zaffaroni, The baryonic branch of Klebanov-Strassler solution: a supersymmetric family of $\mathrm{SU}(3)$ structure backgrounds, JHEP 03 (2005) 069 [hep-th/0412187] [INSPIRE]. 